Schleicher, Andreas (2016). Desafíos para PISA. RELIEVE, 22(1), art. M13. DOI:

\title{
Desafíos para PISA
}

\author{
Challenges for PISA
}

\section{Schleicher, Andreas}

\section{OCDE}

\begin{abstract}
The OECD Programme for International Student Assessment (PISA) provides a framework in which over 80 countries collaborate to build advanced global metrics to assess the knowledge, skills and character attributes of the students. The design of assessments poses major conceptual and technical challenges, as successful learning. Beyond a sound conceptual foundation, PISA needed to fulfil a range of sometimes competing demands. The school administrators, policymakers, and teachers need to be able to use this assessment information to determine how to create better opportunities for student learning. Assessments should also provide productive feedback, at appropriate levels of detail, to fuel improvement and accountability decisions at each level of the education systems. In fact, also to assess of reading, mathematics and science as key foundation skills, PISA is now progressively incorporating also some of the broader cognitive, social and emotional competencies.
\end{abstract}

\section{Keywords:}

PISA; assessment; assessment of educational systems; education; education policy; competences

Reception Date

2016 April 3

\section{Approval Date}

2016 June 14

Publication Date:

2016 June 14

\section{Resumen}

El Programa para la Evaluación Internacional de Alumnos (PISA) proporciona un marco en el que más de 80 países colaboran para construir mediciones globales avanzadas para explorar los conocimientos, habilidades y atributos del estudiantado. El diseño de las evaluaciones plantea importantes desafíos conceptuales y técnicos, como el éxito en el aprendizaje. Más allá de una base conceptual sólida, PISA necesita cumplir una serie de exigencias a veces contradictorias. Los gestores de escuelas, los políticos y los docentes deben ser capaces de utilizar esta información de la evaluación para crear mejores oportunidades para el aprendizaje de los estudiantes. Las evaluaciones también deben proporcionar retroalimentación productiva, con niveles apropiados de detalle, para potenciar las decisiones de mejora y rendición de cuentas, en cada nivel de los sistemas educativos. De hecho, además de evaluar la lectura, las matemáticas y la ciencia como competencias clave, PISA va incorporando progresivamente también algunas competencias más generales de tipo cognitivo, sociales y emocionales.

Fecha de recepción 3 Abril 2016

Fecha de aprobación 14 Junio 2016

Fecha de publicación 14 Junio 2016

\section{Palabras clave:}

PISA; Evaluación; evaluación de sistemas educativos; política educativa; competencias

Las demandas en los sistemas educativos modernos están evolucionando rápidamente. En el pasado, la educación se ocupaba de enseñar algo a la gente. Ahora, se trata de asegurarse de que los estudiantes puedan orientarse y desarrollen competencias para encontrar su propio camino a través de un mundo cada vez más incierto, volátil y ambiguo.
En estos días, ya no se sabe exactamente cómo se desarrollarán las cosas, a menudo nos sorprenden y necesitamos aprender de lo extraordinario, y a veces cometemos errores en el camino. Y frecuentemente serán esos errores y fallos, cuando son comprendidos adecuadamente, lo que cree el contexto para el aprendizaje y la mejora. Hace una generación, los profesores podían pretender que lo que

\section{Autor de contacto / Corresponding author}

Schleicher, Andreas. Directorate for Education and Skills, OECD. 2, rue André Pascal 75775 Paris Cedex 16 (France). (Andreas.SCHLEICHER@oecd.org). 
enseñaban a sus estudiantes les serviría para toda la vida. Hoy en día, las escuelas han de preparar a los estudiantes para unos cambios económicos y sociales más vertiginosos que los que haya habido nunca, para trabajos que aún no existen, para utilizar tecnologías que aún no se han inventado y para resolver problemas sociales que aún no sabemos que van a aparecer.

¿Cómo propiciar estudiantes que estén implicados y motivados para superar los desafíos imprevistos del mañana, por no hablar de los de hoy? El dilema para los educadores es que las habilidades cognitivas rutinarias, las habilidades que son más fáciles de enseñar y de evaluar, son también las capacidades que son más fáciles de digitalizar, automatizar y externalizar. No hay duda de que el conocimiento y las habilidades técnicas en una disciplina seguirán siendo siempre importantes. Las personas innovadoras 0 creativas suelen tener habilidades especializadas en un campo de conocimiento o en un ámbito de aplicación. Y por mucho que las habilidades de "aprender a aprender" sean importantes, aprendemos siempre aprendiendo algún contenido. Sin embargo, el éxito educativo ya no se vincula con la reproducción de conocimientos y contenidos, sino con la extrapolación los que sabemos y aplicándolo a situaciones novedosas. En pocas palabras, el mundo ya no recompensa a las personas sólo por lo que saben -Google o Weibo lo saben todo- sino por lo que pueden hacer con lo que saben. Debido a es esto lo que marca la diferencia en la actualidad, la educación se ocupa cada vez más sobre las formas de pensar (incluyendo creatividad, pensamiento crítico, resolución de problemas y toma de decisiones), sobre los métodos de trabajo (incluida habilidades de comunicación y colaboración); sobre herramientas para trabaja (incluyendo la capacidad de reconocer y explotar el potencial de las nuevas tecnologías) y por último, pero no menos importante, sobre de las habilidades sociales y emocionales que ayudan a las personas a vivir y trabajar juntos (Schleicher, 2012).
Tradicionalmente nuestro abordaje de los problemas consistía en descomponerlos en trozos y en partes manejables, para luego enseñar a los estudiantes técnicas de cómo resolverlos. Pero hoy creamos valor mediante la síntesis de cosas dispares. Esto tiene que ver con la curiosidad, con tener una mente abierta, con hacer conexiones entre ideas que antes parecían no relacionadas entre sí..., todo lo cual requiere estar 'familiarizado con' y 'receptivo a' los conocimientos en otros campos diferentes del propio. Si nos pasamos toda nuestra vida encajonados en un solo ámbito o disciplina, no obtendremos las habilidades imaginativas para conectar los puntos de los que obtendremos la próxima invención.

El mundo ya no se divide entre especialistas y generalistas. Los especialistas generalmente tienen dominio de algunas habilidades y un enfoque reducido, lo que les convierte en expertos reconocidos por sus compañeros, pero que no son valorados fuera de su ámbito. Los generalistas tienen un enfoque amplio pero apenas dominan algunas habilidades en profundidad. Lo que cuenta cada vez más son los "versatilistas" que son capaces de aplicar su pericia sobre un conjunto de situaciones y experiencias cada vez mayor, adquiriendo nuevas competencias, relacionando cosas y asumiendo nuevos roles. Ellos no sólo son capaces de adaptarse constantemente, sino también de un aprender constantemente, de mejorar, de posicionarse y reposicionarse en un mundo en constante cambio.

Quizás más importante aún es el tema de que en las escuelas de hoy en día, los estudiantes aprenden generalmente de forma individual $\mathrm{y}$ al final de cada año escolar certificamos su rendimiento individual. Pero cuanto más interdependiente va siendo el mundo, más nos basamos en grandes coordinadores y colaboradores que sean capaces de relacionarse con los otros en la vida cotidiana, el trabajo y la vida social y política. La innovación, actualmente, rara vez son el producto de individuos que trabajen de forma 
aislada, sino que es un resultado de cómo nos movilizamos, compartimos y relacionamos conocimientos. Las escuelas deben preparar a los estudiantes para un mundo en el que muchas gente tiene que colaborar con personas de diversos orígenes culturales y tomar en consideración ideas, planteamientos y valores diferentes. Un mundo en el que la gente tiene que decidir cómo confiar y colaborar a través de tales diferencias; y un mundo en el que su vida se verá afectada por los problemas que trascienden las fronteras nacionales. Dicho de otra manera, las escuelas necesitan impulsar un cambio de un mundo en el que el conocimiento acumulado deprecia rápidamente su valor, hacia un mundo en el que está aumentando el poder enriquecedor de la comunicación y los flujos de colaboración (Schleicher, 2015).

En muchas escuelas de todo el mundo, los maestros intentar que sus estudiantes desarrollen ese tipo de conocimientos, habilidades y atributos de carácter. Pero los sistemas educativos siguen debatiendo sobre cómo reflejar eso en las evaluaciones y en las pruebas que se utilizan para validar $\mathrm{y}$ reconocer lo que saben y pueden hacer los estudiantes. De hecho, la gran mayoría de las tareas en muchos exámenes tradicionales ahora pueden resolverse con la ayuda de un teléfono inteligente. Si queremos saber si los estudiantes son más inteligentes que un teléfono inteligente, necesitamos construir exámenes y evaluaciones más avanzadas.

\section{El proyecto PISA}

El Programa para la Evaluación Internacional de Alumnos (PISA) de la OCDE proporciona un marco en el que más de 80 países colaboran para construir mediciones globales avanzadas para explorar los conocimientos, habilidades y atributos característicos que son relevantes para el éxito del estudiante y que son esenciales para la plena participación en las sociedades modernas.
El diseño de las evaluaciones plantea importantes desafíos conceptuales y técnicos, en tanto que el aprendizaje exitoso tiene que ver tanto con el procesamiento como con los hechos y datos. El diseño de PISA comenzó con el establecimiento de un marco conceptual que se extendía desde el desarrollo de una definición operativa para los ámbitos de evaluación que deben evaluarse hasta la descripción de los supuestos que subyacen a esa definición; un examen sobre la forma de organizar un conjunto de tareas construidos con el fin de informar a los responsables políticos y a los investigadores sobre el tema del rendimiento en cada área de evaluación; la identificación de un conjunto de características clave que deben tenerse en cuenta a la hora de construir las tareas de evaluación de modo que reflejen de manera significativa las mejoras en el aprendizaje; la puesta en marcha de un conjunto de características clave que se utilizarán en la construcción de pruebas; la validación de las variables y la evaluación de la contribución que hace cada una a la comprensión de la dificultad de la tarea. El marco teórico proporciona la base para el diseño de las tareas que se pueden utilizar para generar información sobre las respuestas de los estudiantes, la codificación/valoración de dichas respuestas, la realización de las tareas y la recogida de información, y el modelado de las respuestas con respecto a las construcciones que deben evaluarse.

Más allá de una base conceptual sólida, PISA necesita cumplir una serie de exigencias a veces contradictorias. La más obvia, que las evaluaciones deben hacerse según los estándares, ser técnicamente sólidas y válidas para su propósito. También tienen que basarse en una serie de métodos para garantizar una medición adecuada de los constructos y discriminar distintos niveles para servir a las necesidades de toma de decisión decisiones en los diferentes niveles de los sistemas educativos. Las evaluaciones también deben proporcionar retroalimentación productiva, con niveles adecuados de detalle, para impulsar las decisiones de mejora y la 
rendición de cuentas en todos los niveles de los sistemas educativos. Los profesores tienen que ser capaces de entender lo que la evaluación revela acerca del pensamiento de los estudiantes. Y los directores de las escuelas, los políticos y los profesores deben ser capaces de utilizar esta información de la evaluación para determinar cómo crear mejores oportunidades para el aprendizaje de los estudiantes. PISA se enfrenta al reto adicional de asegurar que los resultados sean válidos a través de fronteras culturales, lingüísticas y nacionales sobre las que se extienden, y que las poblaciones objetivo de las que se extraen las muestras en los países participantes sean comparables (Schleicher, 2014).

PISA comenzó sus evaluaciones en el año 2000 con una evaluación en profundidad de las destrezas de alfabetización, que se definen como la comprensión, el uso, la reflexión y la implicación con textos escritos. Este concepto fue deliberadamente más allá de la noción tradicional de la decodificación de la información y de la interpretación literal de lo que está escrito, y abarca a la amplia gama de situaciones en las que la gente lee, las diferentes formas en las que se presentan textos escritos a través de diferentes medios de comunicación, y la variedad de formas en las que los lectores utilizan los textos, desde la funcional y finita (como encontrar un trozo concreto de información práctica), a la profunda y de largo alcance (tales como la comprensión de otras formas de hacer, de pensar y de ser). Desde el año 2000, las evaluaciones de lectura de PISA han evolucionado considerablemente, reflejando en gran parte los cambios en la naturaleza de la lectura con el advenimiento de las tecnologías digitales. En el pasado, los profesores debían decir a los estudiantes que buscasen la información perdida en una enciclopedia, y confiar en que esa información, en general, fuese precisa y verdadera. Hoy en día, los textos digitales requieren que los estudiantes gestionen estructuras de información no lineales, construyan su propia representación mental de la información para a través de hipertexto en internet para adaptarlo a su manera, y que hagan frente a la ambigüedad e interpreten $\mathrm{y}$ resuelvan informaciones incongruentes que se encuentran por algún lugar de la web. De hecho, cuantas más tecnologías digitales de contenido de conocimiento permiten a los estudiantes la búsqueda y acceso, más importante se vuelve la capacidad de extraer el significado de ese contenido, y la capacidad de que los estudiantes se cuestionen o intenten mejorar el conocimiento aceptados y las prácticas de cada momento (OECD, 1999).

En 2003, el foco de PISA se dirigió hacia la evaluación de las matemáticas, definidas como la capacidad de los estudiantes para formular, emplear e interpretar las matemáticas en una variedad de contextos. La evaluación versó sobre el razonamiento matemático y el uso de conceptos matemáticos, procedimientos, datos y herramientas para describir, explicar y predecir fenómenos. También se les pidió a los estudiantes demostrar que podían reconocer el papel de las matemáticas en el mundo y realizar los juicios de manera bien fundamentada y tomar el tipo de decisiones que necesitan los ciudadanos constructivos, comprometidos y reflexivos. Este enfoque se fundamenta en la importancia de las matemáticas para una participación plena en la sociedad y se considera que esta importancia se debe a la forma en que las matemáticas puedan ser usadas para describir, explicar y predecir fenómenos de muchos tipos. PISA ha establecido un conjunto de siete capacidades matemáticas fundamentales que sustentan el desempeño en las evaluaciones de matemáticas de PISA: (1) La comunicación es tanto receptiva y expresiva. La lectura, la decodificación e interpretación de las planteamientos, preguntas, tareas u objetos permiten a la persona formar un modelo mental de la situación. Además, el que resuelve un problema puede que tenga que presentar o explicar la solución. "Matematizar" consiste en saber saltar entre el mundo real y el mundo matemático. Tiene dos 
partes: formulación e interpretación. Formular un problema como un problema matemático puede incluir estructuración, conceptualización, plantear suposiciones y/o construir un modelo. La interpretación consiste en determinar si los resultados de la resolución matemática se relacionan con el problema original y juzgar su idoneidad. (3) La representación implica seleccionar, interpretar, traducir y usar una variedad de representaciones para capturar una situación, interactuar con un problema, o presentar el modo de funcionamiento propio. (4) Se requiere razonar y la argumentar a lo largo de las diferentes etapas y actividades asociadas con la competencia matemática. Esta capacidad implica procesos de pensamiento arraigados en la lógica de explorar y enlazar elementos del problemas con el fin de ser capaz de realizar inferencias a partir de ellos, comprobar una justificación que se haya dado, o proporcionar una justificación de planteamientos o de soluciones a los problemas. (5) Elaborar de estrategias para la resolución de problemas se plantea como la selección o elaboración de un plan o estrategia para usar las matemáticas a fin de resolver problemas derivados de una tarea o contexto, y guiar y supervisar su aplicación. (6) Usar un lenguaje y operaciones simbólicas, formales y técnicas lo que implica comprender, interpretar, manipular y hacer uso de las expresiones $\mathrm{y}$ operaciones aritméticas $\mathrm{y}$ simbólicas, usar construcciones formales basadas en las definiciones, las normas y los sistemas formales, y usar algoritmos en esos casos. Por último, (7), utilizar herramientas matemáticas implica conocer y ser capaz de utilizar varias herramientas (físicas o digitales) que puedan ayudar a la actividad matemática, y conocer las limitaciones de este tipo de herramientas (OECD, 2003).

En 2006, el foco de PISA se desplazó hacia la ciencia, que se define como la capacidad de los estudiantes a implicarse con temas relacionadas con la ciencia y con las ideas de la ciencia, como un ciudadano reflexivo. Para hacerlo bien en la prueba de ciencias de PISA, los estudiantes necesitan estar dispuestos a seguir un discurso razonado acerca de la ciencia y la tecnología. Esto requiere que las competencias para (1) explicar fenómenos científicamente (lo que implica la capacidad de reconocer, plantear y evaluar explicaciones para una serie de fenómenos naturales $\mathrm{y}$ tecnológicos), (2) Evaluar y diseñar investigación científica (lo que implica la capacidad de describir y evaluar investigaciones científicas y proponer formas de abordar las cuestiones científicamente) y (3) interpretar los datos y evidencias científicamente (lo que implica la capacidad de analizar y evaluar datos, planteamientos y argumentos en una variedad de presentaciones, sacando la conclusión científica apropiada). Explicar los fenómenos científicos y tecnológicos exige un conocimiento del contenido de la ciencia. La segunda y tercera competencia, sin embargo, requieren más que un conocimiento de lo que sabemos. Más bien, dependen de una comprensión de cómo se establece el conocimiento científico y del grado de confianza con el que mantiene. Reconocer e identificar los rasgos que caracterizan la investigación científica requiere un conocimiento de los procedimientos que subyacen a los diversos métodos y prácticas utilizadas para establecer el conocimiento científico - que se denomina en este caso como conocimiento procedimental-. Por último, las competencias requieren un conocimiento epistémico -una comprensión de los fundamentos de las prácticas habituales de la investigación científica, el estatus de las afirmaciones que se generan y el significado de los términos fundamentales, tales como teoría, hipótesis y datos- (OECD, 2006).

\section{Desafíos para el proyecto PISA}

Si bien PISA continúa con la evaluación de la lectura, las matemáticas y la ciencia como competencias clave de cimentación, ahora actualmente está incorporando progresivamente también algunas de las competencias cognitivas, sociales y emocionales más amplias comentadas anteriormente. La evaluación de las 
competencias sociales se ha convertido en una prioridad en el año 2015. Como se ha señalado antes, los jóvenes que pasan a integrarse como población activa y en la vida pública necesitan habilidades y actitudes para colaborar y resolver eficazmente los problemas, cada vez más en situaciones en las que los miembros del grupo están geográficamente dispersos, trabajan en diferentes zonas horarias, y se conectan a través de la tecnología. Las sociedades esperan de ellos que tengan la capacidad de resolver problemas y proporcionar soluciones colaborativamente a través de la puesta en común de conocimientos, habilidades y esfuerzos. Como primer paso, PISA introdujo en 2015 la evaluación de las habilidades de resolución colaborativa de problemas, que evalúa a los estudiantes en función de tres competencias básicas: (1) establecer y mantener una comprensión compartida; (2) adoptar medidas apropiadas para resolver problemas; y (3) establecer y mantener la organización del equipo. Para facilitar esta tarea, se requiere que los estudiantes interactúen y colaboren con los miembros del equipo generado por ordenador(s) en situaciones controladas para resolver un problema particular. Este proceso requiere que los estudiantes determinen su propio papel y sus responsabilidades en lo que respecta a otros agentes, el seguimiento de los aspectos de la organización del grupo, y facilitar los ajustes y cambios que se necesiten cuando la comunicación se rompa, cuando aparecen nuevos obstáculos o cuando se presenten oportunidades para la optimización del rendimiento.

Por otro lado, los países están actualmente colaborando para desarrollar una evaluación de la competencia global que se incluirá en 2018 y que se define como la capacidad y disposición para actuar e interactuar de manera adecuada y efectiva, tanto de forma individual como en equipo, participando en un mundo interconectado, interdependiente y diverso. La evaluación se basa en cuatro aspectos fundamentales: (1) La comunicación y la gestión de relaciones -que se refiere a la disposición y la capacidad de adaptar comunicación y el comportamiento de cada cual con el fin de interactuar de manera adecuada y eficaz con otros que mantienen perspectivas diversas, en diferentes contextos. (2) El conocimiento y el interés por los desarrollos, retos y tendencias globales -que se refiere a los intereses del alumno y el conocimiento de las culturas, las cuestiones, eventos y fenómenos importantes en el mundo, así como la capacidad del alumno para comprender su importancia global y sus implicaciones para adapta de manera adecuada y eficaz al aprendizaje, al trabajo, y situaciones con otras personas que mantienen perspectivas distintas y en diferentes contextos. (3) La apertura y flexibilidad -que se refiere a la receptividad y comprensión de nuevas ideas, personas y situaciones, así como a de diferentes perspectivas y prácticas. También se refiere a la capacidad de buscar y comprender perspectivas y experiencias nuevas y diferentes de manera adecuada y adaptar de manera apropiada y eficaz la propia manera de pensar, los comportamientos y actividades de aprendizaje, de trabajo y de vida cotidiana que involucran a otros que mantienen perspectivas distintas y en diferentes contextos. (4) La fuerza emocional y la resiliencia -que se refiere a la capacidad, al desarrollo de mecanismos de afrontamiento y resiliencia, para tratar de forma apropiada con la ambigüedad, los cambios y desafíos que esas perspectivas y experiencias diferentes pueden presentar-.

A medida que nuestras sociedades vayan evolucionando más, los países participantes en PISA continuarán colaborando para definir y medir los conocimientos, habilidades y atributos del carácter que ayudarán a las personas a mantenerse al tanto de estos cambios para satisfacer las crecientes demandas. Cada tres años, los resultados de PISA proporcionan una herramienta poderosa que los países pueden utilizar para desarrollar, revisar y ajustar sus estándares curriculares y las políticas educativas, con el objetivo de proporcionar la mejor educación posible para todos sus estudiantes. La OCDE está dispuesta a apoyar a los países en esta tarea desafiante y crucial. 
Schleicher, Andreas (2016). Desafíos para PISA. RELIEVE, 22(1), art. M13. DOI:

\section{Referencias}

OECD. (1999). Measuring student knowledge and skills. A new Framework for assessment. Paris: OECD. Consultado en http://www.oecd.org/edu/school/programmefo rinternationalstudentassessmentpisa/33693997. pdf

OECD. (2003). The PISA 2003. Assessment Framework. Mathematics, Reading, Science and Problem Solving knowledge and skills. Paris: OECD. Consultado en http://www.oecd.org/edu/school/programmefo rinternationalstudentassessmentpisa/33694881. pdf

OECD. (2006). Assessing Scientific, Reading and Mathematical Literacy. A Framework for PISA 2006. Paris: OECD. Consultado en http://www.oecdilibrary.org/docserver/downlo ad/9806031e.pdf?expires $=1465551043 \& i d=i d$ \&accname=guest \&checksum=02E5A7F7B73 F1CCFA0DA6E8336A2F3D8

Schleicher, A. (2012). Preparing Teachers and Developing School Leaders for the 21st Century: Lessons from around the World. Paris: OECD Publishing. doi: http://dx.doi.org/10.1787/9789264xxxxxx-en

Schleicher, A. (2014). Equity, Excellence and Inclusiveness in Education Policy Lessons from Around the World. Paris: OECD Publishing. Consultado en https://www.ucy.ac.cy/equality/documents/Art icles-Material/OECD_2014_Report.pdf

Schleicher, A. (2015). Schools for 21st-Century Learners: Strong Leaders, Confident Teachers, Innovative Approaches, International Summit on the Teaching Profession. Paris: OECD Publishing. doi: http://dx.doi.org/10.1787/9789264231191-en.

\section{Author / Autor}

To know more / Saber más

Schleicher, Andreas (Andreas.SCHLEICHER@oecd.org).

Director de Educación y Capacitación, y Asesor Especial sobre Políticas de Educación para el Secretario General de la Organización para la Cooperación y el Desarrollo (OCDE) en París. Su labor en la Dirección incluye el Programa para la Evaluación Internacional de Alumnos (PISA), el estudio de competencias de los adultos (PIAAC), y la Encuesta Internacional de Aprendizaje (TALIS), y el desarrollo y análisis de los indicadores sobre el rendimiento de los sistemas educativos (INES). Antes de incorporarse a la OCDE, fue Director de Análisis de la Asociación Internacional para el Rendimiento Educativo (IEA). Se licenció en Física en Alemania y obtuvo un título en Matemáticas y Estadística en Australia. Su dirección postal es: Directorate for Education and Skills, OECD ,2, rue André Pascal 75775 Paris Cedex 16 (Francia).

\section{RELIEVE}

\section{Revista ELectrónica de Investigación y EValuación Educativa E-Journal of Educational Research, Assessment and Evaluation [ISSN: 1134-4032]}

\footnotetext{
(C) Copyright, RELIEVE. Reproduction and distribution of this articles it is authorized if the content is no modified and their origin is indicated (RELIEVE Journal, volume, number and electronic address of the document).

(C) Copyright, RELIEVE. Se autoriza la reproducción y distribución de este artículo siempre que no se modifique el contenido y se indique su origen (RELIEVE, volumen, número y dirección electrónica del documento).
} 
